\title{
The influence of distraction on speech processing: How selective is selective attention?
}

\author{
Sandra I. Parhammer ${ }^{1,2 *}$, Miriam Ebersberg ${ }^{1,3^{*}}$, Jenny Tippmann ${ }^{4 *}$, Katja Stärk $^{5 *}$, Andreas Opitz ${ }^{6}$, \\ Barbara Hinger ${ }^{2}$, and Sonja Rossi ${ }^{1}$ \\ ${ }^{1}$ ICONE - Innsbruck Cognitive Neuroscience, Department for Hearing, Speech, and Voice \\ Disorders, Medical University of Innsbruck, Austria \\ ${ }^{2}$ Department of Subject-Specific Education, Area of Language Education, \\ University of Innsbruck, Austria \\ ${ }^{3}$ Department of Psychology, University of Innsbruck, Austria \\ ${ }^{4}$ Department of Child and Adolescent Psychiatry and Psychotherapy, \\ Technical University of Dresden, Germany \\ ${ }^{5}$ Language Development Department, Max-Planck-Institute for Psycholinguistics, \\ Nijmegen, The Netherlands \\ ${ }^{6}$ Herder-Institute, University of Leipzig, Germany \\ sonja.rossi@i-med.ac.at
}

\begin{abstract}
The present study investigated the effects of selective attention on the processing of morphosyntactic errors in unattended parts of speech. Two groups of German native (L1) speakers participated in the present study. Participants listened to sentences in which irregular verbs were manipulated in three different conditions (correct, incorrect but attested ablaut pattern, incorrect and crosslinguistically unattested ablaut pattern). In order to track fast dynamic neural reactions to the stimuli, electroencephalography was used. After each sentence, participants in Experiment 1 performed a semantic judgement task, which deliberately distracted the participants from the syntactic manipulations and directed their attention to the semantic content of the sentence. In Experiment 2, participants carried out a syntactic judgement task, which put their attention on the critical stimuli. The use of two different attentional tasks allowed for investigating the impact of selective attention on speech processing and whether morphosyntatic processing steps are performed automatically. In Experiment 2, the incorrect attested condition elicited a larger N400 component compared to the correct condition, whereas in Experiment 1 no differences between conditions were found. These results suggest that the processing of morphosyntactic violations in irregular verbs is not entirely automatic but seems to be strongly affected by selective attention.
\end{abstract}

Index Terms: auditory speech perception, selective attention, morphosyntax, electroencephalography

\section{Introduction}

Listening to lectures, having telephone conversations, understanding a conversational partner - for all of these tasks the ability to extract important information from connected

*These authors contributed equally. auditory speech input, which is an integral part of the human speech comprehension system, is indispensable. Previous studies have shown that adult L1 speakers can easily filter out relevant information from auditory speech input, whereas children still have to develop such advanced auditory discrimination abilities [1], [2], [3]. How selective is selective attention? And is morphosyntactic information in unattended parts of speech even processed by the brain or do errors in unattended parts go completely unnoticed?

Investigating event-related brain potentials (ERPs) in the brain allows for identifying fast dynamic changes in the range of milliseconds. Therefore, this neuroscientific research method is especially suited for the investigation of automatic speech processing, as it occurs very fast and often prior to conscious perception. Previous studies have shown that syntactic errors in sentences elicit a biphasic ERP pattern in monolingual adult listeners: an (early) left anterior negativity (E-LAN) and a P600 (e.g. [4], [5], [6], [7], [8], [9], [10]). The former is associated with the detection of (morpho-)syntactic errors, while the latter is suggested to reflect syntactic repair or reanalysis processes. Yet, this pattern was found to be only elicited if participants directly attend to the syntactic violations under investigation. Some ERP studies explicitly investigated the automaticity of these processing steps by directing the participants' attention away from the relevant linguistic input and found diverging results: either an absence or reduction in amplitude of ERP components or the emergence of different processing mechanisms [1], [3], [11]. All these results support the assumption that attention plays a crucial role during syntactic processing. None of these studies, however, specifically looked at syntactic violations with different degrees of severity. Therefore, the question remains whether the severity of violations may also have an influence on the degree of automaticity of language processing.

Regel, Opitz, Müller and Friederici [12] addressed this issue by investigating visually presented, morphosyntactically 
manipulated German irregular verbs embedded in minimal syntactic contexts (i.e. phrases). They found a biphasic LANP600 pattern of which the amplitude of the latter was modulated by the severity of the violations. In fact, the P600 amplitude was larger for the irregular verbs following a crosslinguistically unattested ablaut pattern than for irregular verbs following an attested ablaut pattern of German verb inflection. Attention to the stimuli was ensured by means of a judgement of the correctness of the phrase, which explicitly directed the participants' attention to the critical stimuli. The question arises whether the results would have been different if another attentional task had been used.

The overall aim of the present study was to investigate the neural processing of morphosyntactic errors in unattended speech. Two participant groups took part in one of two almost identical experiments, which only differed in the attentional task.

As the incorrect unattested condition used by Regel et al. [12] reflects a pattern which is dispreferred across many languages (cf. [13], [14]), this condition represented the most severe violation. Therefore, it is possible that such an unnatural pattern could be recognized and processed automatically even if it is located in the unattended parts of a sentence. To further investigate this, we used a selection of the manipulated irregular verbs utilized by Regel et al. (2015) and presented them in otherwise syntactically and semantically correct sentences in German applying two different types of attentional tasks (a semantic judgement task diverting the attention away from the critical stimuli and a syntactic judgement task drawing the attention to the critical stimuli). To further examine whether the severity of the violations influences automatic speech processing, irregular verbs appearing in three different conditions were used in the present study: the correct past tense form, an incorrect past tense form with an attested ablaut pattern, and an incorrect past tense form following a crosslinguistically unattested ablaut pattern (cf. Tab. 1).

\section{Methods}

The present study was conducted at the Medical University of Innsbruck in cooperation with the University of Leipzig. The study was authorized by the ethics committee of the Medical University of Innsbruck and written informed consent was obtained from each participant.

\subsection{Participants}

Two groups of healthy adult German native speakers participated in the experiments. The participants of both groups were right-handed according to the Edinburgh Inventory of Handedness [15], were raised monolingually, were not born prematurely, did not have any known hearing deficits, had a normal or corrected-to-normal vision, and did not receive any monetary compensation. The group of Experiment 1 consisted of 26 participants; however, one participant had to be excluded from analyses due to a developmental language disorder. The remaining 25 participants ( 14 females) were between 19 and 29 years of age (mean age: 23.9 years, $\mathrm{SD}=2.95$ ). The group of Experiment 2 consisted of 32 participants. One participant had to be excluded from analyses due to having too many artifacts in the EEG recording. The remaining 31 participants were between 18 and 28 years old (mean age: $23.6, \mathrm{SD}=4.92$ ) and 15 were female.

\subsection{Stimulus material}

The stimulus material was identical in Experiment 1 and 2 and consisted of 78 acoustically presented experimental sentences. The verbs were systematically manipulated resulting in the following three conditions: the CORRECT version of an irregular verb in the past tense (ABC ablaut pattern), an irregular verb following an incorrect but ATTESTED past tense ablaut pattern (ABB ablaut pattern), and an irregular verb with an incorrect and UNATTESTED ablaut pattern (ABA ablaut pattern). The ABA pattern is not used in the German past tense formation and is generally dispreferred across languages [13], [14]. The capital letters (ABC, ABB, ABA) represent the vowel alternations occurring in the three German primary tense stems in the following order: the present tense stem, past participle stem, and past tense stem. For example, AAA symbolizes that all three stems have the same stem vowel, which is the case for regular verbs (e.g. tanzen, getanzt, tanzte; dance, danced, danced). $\mathrm{ABC}$, on the other hand, indicates that the vowel alternates with every primary tense stem (e.g. singen, gesungen, sang; sing, sung, sang). In total, 13 different irregular verbs were used, all of which followed an $\mathrm{ABC}$ ablaut pattern. Two sentences were created with each verb resulting in 26 sentences per condition (cf. Tab. 1). To give an example, the German verb trinken (to drink) appeared in the following sentences:

Table 1: Examples of sentences used in the three experimental conditions.

\begin{tabular}{ll}
\hline Condition & $\begin{array}{l}\text { Example Sentence } \\
\text { (incl. English Translation) }\end{array}$ \\
\hline CORRECT - & Letzten Monat trank der \\
ABC ablaut pattern & Onkel einen starken Kaffee. \\
& Last month the uncle drank \\
a strong coffee.
\end{tabular}

\section{Incorrect ATTESTED ablaut pattern - ABB}

Incorrect UNATTESTED
ablaut pattern - ABA

In addition to these 78 sentences, 26 filler sentences with correct irregular verbs following either an $\mathrm{ABB}$ or $\mathrm{AAB}$ ablaut pattern were used in the experiment to balance the number of incorrect and correct sentences. Thus, in total, the experiment comprised 104 items.

Apart from the morphosyntactic violations in the verbs, the sentences were correct and all followed the same syntactic structure: adverbial phrase - verb - subject - object. The past tense forms of all verbs used in the experiment were monosyllabic, irregular, transitive, and non-reflexive. The subject always consisted of a monosyllabic definite article and a disyllabic noun and all objects consisted of a monosyllabic indefinite article, a disyllabic adjective, and a disyllabic noun. All sentences were recorded by a female German native speech scientist with 16 bits and a sampling rate of $48 \mathrm{kHz}$. 


\subsection{Experimental procedure}

During the experiment, the participants were seated approximately $70 \mathrm{~cm}$ in front of a computer monitor and listened to sentences that were acoustically presented via stereo speakers at a volume of $70 \mathrm{~dB}$. Every trial started with an initial $1000 \mathrm{~ms}$ of silence, subsequently the auditory presentation of the sentence was played for $4219 \mathrm{~ms}$ on average $(M I N=3631$ $\mathrm{ms}, M A X=5024 \mathrm{~ms}, S D=240.55 \mathrm{~ms}$ ) followed by another $3000 \mathrm{~ms}$ of silence. During all of this, a fixation cross was displayed on a computer screen.

In Experiment 1 (semantic judgement task), after the 3000 ms of silence a picture (taken from Rossion \& Pourtois [16] as well as from license-free online databases) appeared for a maximum of $5000 \mathrm{~ms}$. During this time, the participants had to decide whether the object displayed on the screen had been mentioned in the previous sentence or not by pressing a button (green or red; green indicated that it had been part of the sentence). Once the participants clicked on the button, the next trial started automatically and the display switched back to the fixation cross. The position of the green and red button (right or left click) was counterbalanced across participants. The semantic judgement task deliberately directed the participants' attention away from the syntactic (in)correctness of the critical verbs and to the semantic content of the nouns, which allowed for an investigation of whether or not morphosyntactic errors are even noticed in unattended parts of speech.

In Experiment 2 (syntactic judgement task), the stimulus material was identical; however, instead of the semantic judgement task a syntactic judgement task was used. During this task a smiling (for syntactically correct sentences) and a sad emoticon (for syntactically incorrect sentences) were presented for a maximum of $5000 \mathrm{~ms}$. Participants had to decide whether a sentence was correct by clicking the button on the side of the corresponding emoticon, the position of which was counterbalanced across participants.

\subsection{EEG recordings and data analyses}

The electroencephalogram (EEG) was recorded from 34 AgAgCI electrodes (BrainProducts GmbH, Gilching, Germany), which were integrated in a flexible EEG cap (EasyCap, Herrsching, Germany). The following electrode positions were used for the recording: F3, F7, FC3, FT7, C3, T7, CP3, CP5, P3, P7, PO3, O1, Fz, FCz, Cz, CPz, Pz, F4, F8, FC4, FT8, C4, T8, CP4, CP6, P4, P8, PO4, and O2. During the recording, the EEG was referenced to an online common reference placed on the left mastoid (TP9) and was later rereferenced to the average of left and right mastoids (TP10) during the analysis of the EEG recording. AFz served as the ground electrode and the electrooculogram (EOG) was recorded from the electrodes FP2 (horizontal) and F10 (vertical), which was used to correct artifacts from blinking and eye movements. The impedance was kept below $10 \mathrm{k} \Omega$. Before the EEG signal was digitized, it was filtered online with an analog/digital converter with an upper cut-off of $450 \mathrm{~Hz}(24$ $\mathrm{dB} / \mathrm{Oct}$ ) to prevent aliasing. The recording was digitized online with a sampling rate of $1000 \mathrm{~Hz}$.

The data was prepared for statistical analyses in several steps. The EEG recording only contained marker files for the onset of each sentence. Thus, a script was programmed in MATLAB R2015a, with which the exact position of the critical verb within each sentence could be determined in the EEG recording. A low-pass filter of $30 \mathrm{~Hz}$ was applied to the EEG recording. Then the signal was segmented from -200 to 1500 $\mathrm{ms}$ based on the onset of the critical verb. Ocular artifacts were removed by means of an algorithm based on Gratton, Coles, and Donchin [17]. Afterwards, other, non-cerebral artifacts were removed from the recording manually. Subsequently, a prestimulus-onset baseline correction from -200 to $0 \mathrm{~ms}$ was applied. Finally, single-subject averages were calculated across all trials per condition and the grand averages were computed.

For the final statistical analyses three time windows were selected based on a visual inspection of the grand averages and a 50-ms-analysis: $100-300 \mathrm{~ms}, 300-900 \mathrm{~ms}$, and $900-1300 \mathrm{~ms}$. For each experiment (semantic and syntactic judgement task) repeated measure ANOVAs including the within-subject factors condition (correct, incorrect attested, incorrect unattested), region (inferior frontal, superior frontal, temporal, centroparietal, parietal) and hemisphere (left, right) were computed for 10 different regions of interest (ROIs): F7FT7, F8FT8, F3FC3, F4FC4, T7CP5, T8CP6, C3CP3, C4CP4, $\mathrm{P} 3 \mathrm{PO} 3, \mathrm{P} 4 \mathrm{PO} 4$. The midline electrodes (Fz, $\mathrm{FCz}, \mathrm{Cz}, \mathrm{CPz}, \mathrm{Pz}$ ) were analyzed separately in an ANOVA condition $\mathrm{x}$ electrodes. A significance level of $p<.050$ was applied. Whenever significant main effects of condition or a significant interaction between condition and hemisphere and/or region/electrodes were found, post-hoc $t$-tests were performed and a Bonferroni correction was applied.

\section{Results}

\subsection{Experiment 1 - Semantic Judgement Task}

Statistical analyses for the 100-300 ms time window showed a significant interaction of condition and electrodes $\left(F_{8,192}=3.12\right.$, $p=.034$ ) for the midline electrodes; however, post-hoc $t$-tests did not show any significant differences. No significant effect was found for the time window $300-900 \mathrm{~ms}$. The statistical analysis of the 900-1300 ms time window revealed a significant interaction of condition $\mathrm{x}$ region $\left(F_{8,192}=3.16, p=.019\right)$ for lateral ROIs. However, post-hoc $t$-tests did not show any significant difference. See Fig. 1.

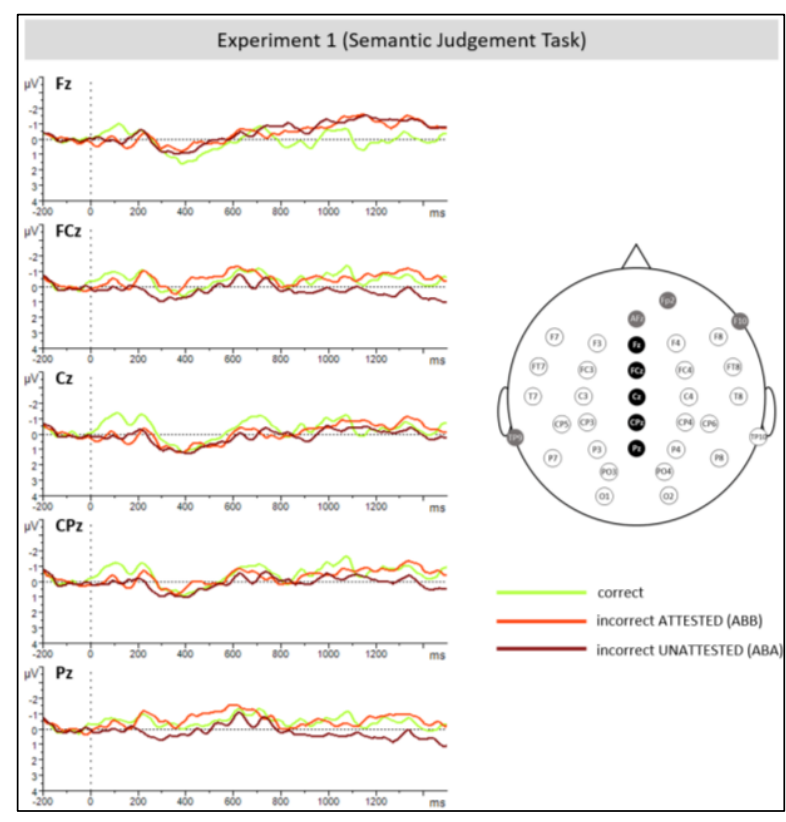

Figure 1: Grand averages of the three experimental conditions at midline electrodes for Experiment 1. Negative voltage is plotted upwards. 


\subsection{Experiment 2 - Syntactic Judgement Task}

The early time window $100-300 \mathrm{~ms}$ did not reveal any significant effects. In the $300-900 \mathrm{~ms}$ time window a significant main effect for the factor condition was found at lateral $\left(F_{2,60}=4.88, p=.011\right)$ as well as midline electrodes $\left(F_{2,60}=3.38\right.$, $p=.041$ ). Post-hoc $t$-tests confirmed a broadly distributed negativity which was significantly larger in amplitude for the incorrect attested verbs relative to the correct condition over all ROIs $\left(t_{30}=3.16, p=.004\right)$ and all midline electrodes $\left(t_{30}=2.57\right.$, $p=.015$ ) resembling an N400-like component. Between 900$1300 \mathrm{~ms}$ a highly significant interaction between condition and electrodes at midline electrodes was found $\left(F_{8,240}=4.27\right.$, $p=.001)$. Post hoc testing confirmed a negativity for the incorrect attested relative to the correct condition $\left(t_{30}=2.18\right.$, $p=.003$ ) at the Fz electrode. See Fig. 2.

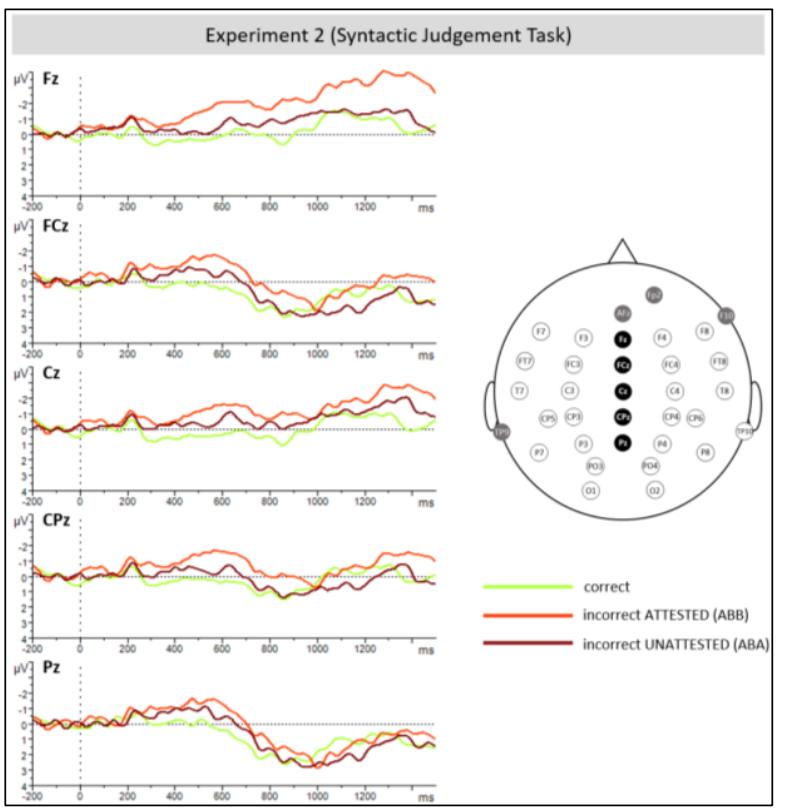

Figure 2: Grand averages of the three experimental conditions at midline electrodes for Experiment 2. Negative voltage is plotted upwards.

\section{Discussion}

The present study investigated the influence of attention on the processing of morphosyntactic errors with different degrees of severity by assessing the automaticity of the neural processing of German irregular verbs.

In Experiment 1, in which the subject's attention was explicitly directed towards the semantic content of the nouns and, thus, away from the syntactic errors, no differences in ERP components were observed between conditions. This indicates that no processing step (i.e. LAN, P600, or N400) seems to be automatic to such a degree that it is elicited irrespective of selective attention.

In contrast to Experiment 1, the results of Experiment 2, in which a syntactic judgement task was used that directly drew the participants' attention to the critical verbs and, thus, triggered the subjects to carry out a syntactic evaluation, revealed significant ERP results. While the effect we found in our study was a broadly distributed negativity similar to an N400, the ERP components observed in similar studies by Regel et al. [12], [18] were a biphasic LAN-P600 pattern. The latter was clearly associated with syntactic processes; however, our results indicate a different neural processing. Regel et al. [12], [18] integrated their morphosyntactic errors in short phrases and presented the whole ablaut pattern to all subjects, which explicitly prompted a pure syntactic analysis. In contrast, the errors used in the present experiments were integrated in natural sentences, which did not present the whole ablaut pattern. Furthermore, the verbs in our sentences required two subsequent arguments, a subject and an object. For such an analysis of argument structure both syntactic but also lexicosemantic processes are necessary. This was indexed by an $\mathrm{N} 400$, which is a reaction that was also observed in previous studies (e.g. [19]). Interestingly, this processing mechanism was only found for the incorrect attested but not for the unattested ablaut pattern.

The comparison between our two experiments clearly indicates that irregular verb processing might not elicit automatic processes per se, as an attentional focus on the experimental manipulation is necessary to launch the previously described neural speech processing mechanisms. This is in line with previous findings showing either no ERP components or reduced ERP amplitudes during the syntactic processing of unattended parts of speech [1], [2], [3], [11]. Our results provide new evidence that attentional focus has an impact on the processing of irregular verbs. In addition, attention seems to differently influence the processing of morphosyntactic errors depending on their degree of severity. These findings highlight the interconnectedness of selective attention and speech processing and open up a discussion on whether a deficit in selective attention might also have an impact on a variety of language functions.

\section{Acknowledgements}

We would like to express our deepest gratitude to Franziska Stephan for recording the auditory stimuli, to Bettina Johst for programming the experiment, and to Josef Seebacher for coding a script for inserting the exact timing of markers from the audio into the EEG file.

\section{References}

[1] T. C. Gunter and A. D. Friederici, "Concerning the automaticity of syntactic processing," Psychophysiology, vol. 36, no. 1, pp. 126-137, 1999.

[2] J. L. Mueller, A. D. Friederici, and C. Männel, "Auditory perception at the root of language learning," Proceedings of the National Academy of Sciences, vol. 109, no. 39, pp. 15953 $15958,2012$.

[3] M. W. F. T. Verhees, D. J. Chwilla, J. Trompand, and C. T. W. M. Vissers, "Contributions of emotional state and attention to the processing of syntactic agreement errors: Evidence from P600," Frontiers in Psychology, vol. 6, no. 388, pp. 1-18, 2015.

[4] T. F. Münte, M. Matzke, and S. Johannes, "Brain activity associated with syntactic incongruencies in words and pseudowords," Journal of Cognitive Neuroscience, vol. 9, pp. 318-329, 1997.

[5] S. Coulson, J. W. King, and M. Kutas, "Expect the unexpected: Event-related brain response to morphosyntactic violations," Language and Cognitive Processes, vol. 13, pp. 21-58, 1998.

[6] L. Osterhout and P. Hagoort, ,A superficial resemblance does not necessarily mean you are part of the family: Counterarguments to Coulson, King and Kutas (1998) in the P600/SPS-P300 debate," Language and Cognitive Processes, vol. 14, pp. 1-14, 1999.

[7] A.D. Friederici, "Towards a neural basis of auditory sentence processing," Trends in Cognitive Sciences, vol. 6, no. 2, pp. 7884, 2002. 
[8] H. Barber and M. Carreiras, "Grammatical gender and number agreement in Spanish: An ERP comparison," Journal of Cognitive Neuroscience, vol. 17, no. 4, pp. 137-153, 2005.

[9] S. Rossi, M. F. Gugler, A. Hahne, and A. D. Friederici, "When word category information encounters morphosyntax: An ERP study," Neuroscience Letters, vol. 384, no. 3, pp. 228-233, 2005.

[10] M. Lück, A. Hahne, and H. Clahsen, "Brain potentials to morphologically complex words during listening," Brain Research, vol. 1077, no. 1, pp. 144-152, 2006.

[11] A. Hahne and A. D. Friederici, "Differential task effects on semantic and syntactic processes as revealed by ERPs," Cognitive Brain Research, vol. 13, no. 3, pp. 339-356, 2002.

[12] S. Regel, A. Opitz, G. Müller, and A. D. Friederici, "The Past Tense Debate revisited: Electrophysiological evidence for subregularities of irregular verb inflection," Journal of Cognitive Neuroscience, vol. 27, no. 9, pp. 1870-1885, 2015.

[13] J. D. Bobaljik, Universals in comparative morphology: Suppletion, superlatives, and the structure of words. Cambridge, MA: MIT Press, 2012

[14] B. Wiese, "Form and function of verbal ablaut in contemporary standard German," in Explorations in integrational linguistics: Four essays on German, French, and Guarani, R. Sackmann, Ed. Amsterdam, Holland: John Benjamins, 2008, pp. 97-158.

[15] R. C. Oldfield, "The assessment and analysis of handedness: The Edinburgh Inventory," Neuropsychologia, vol. 9, no. 1, pp. 97 113, 1971.

[16] B. Rossion and G. Pourtois, "Revising Snodgrass and Vanderwart's Object Pictorial Set: The role of surface detail in basic-level object recognition," Perception, vol. 33, no. 2, pp. 217-236, 2004

[17] G. Gratton, M. G. Coles, and E. Donchin, "A new method for offline removal of ocular artifact," Electroencephalography and Clinical Neurophysiology, vol. 55, no. 4, pp. 468-484, 1983.

[18] S. Regel, S. A. Kotz, I. Henseler, and A. D. Friederici, "Left inferior frontal gyrus mediates morphosyntax: ERP evidence from verb processing in left-hemisphere damaged patients," Cortex, vol. 86, pp. 156-171, 2017.

[19] S. Frisch, A. Hahne, and A. D. Friederici, "Word category and verb-argument structure information in the dynamics of parsing," Cognition, vol. 91, pp. 191-219, 2004. 